\section{Ever-decreasing circles}

It's generally accepted that the single bacterial chromosome of Escherichia coli is organized into approximately 100 equal-sized domains of about 50-100 kb. However, Postow et al. now report in Genes and Development that the chromosome is packed into small, variable domains, with an average size of just $10 \mathrm{~kb}$, that are randomly positioned around the chromosome.

Bacterial chromosomes are condensed about 1,000-fold, in part by DNA gyrase which introduces negative supercoils, so that they fit inside the cell. Supercoiled DNA is arranged into domains and stabilized by proteins and possibly RNAs, so that one strand break doesn't unwind the whole chromosome, which would kill the cell.

By inhibiting DNA gyrase, more than 300 supercoiling-sensitive genes (SSGs) were identified in E. coli. Postow et al. realized that monitoring the expression of the SSG gene-set might allow chromosome domain size to be accurately delineated. They used an inducible restriction enzyme to randomly cut the chromosome, then monitored levels of SSG transcripts using microarrays. The distance from random cut sites to the domain boundary will affect the extent of local unwinding (relaxation) of the supercoils, so that the level of an SSG transcript will only change if the cut site is in the same domain as the SSG. Differences in SSG transcript abundance were detected well before the chromosome was completely degraded - proving that the method works. Expression of individual SSGs was quantitated using RNase protection. The results were compared with Monte Carlo simulations of different models of domain organization and closely matched a model in which the chromosome is packed into randomly placed domains with an average size of 9-11 kb. Gentle chromosome preparation and analysis with electron microscopy confirmed these experimental observations - domains are much smaller than was previously thought.

Domain barriers could be formed by association with the cell membrane, other parts of the chromosome or with macromolecular complexes such as DNA or RNA polymerases. As the domain size is already known to increase when the cell enters stationary phase and global transcript levels decrease, formation of transient domains dependent on local DNA activity is an appealing model.

The domain size determines chromosome compaction, which affects DNA repair, transcription and replication. Small domains are more easily repaired because loose ends from breaks aren't too far apart. As domains form the basis of chromosome organization from bacteria to man, understanding the organization of the E. coli chromosome is relevant to all of us.

Susan Jones Susan Jone
links ORIGINAL RESEARCH PAPER Postow, L. et al. Topological domain structure of the Escherichia coli chromosome. Genes Dev. 18, 1766-1779 (2004)

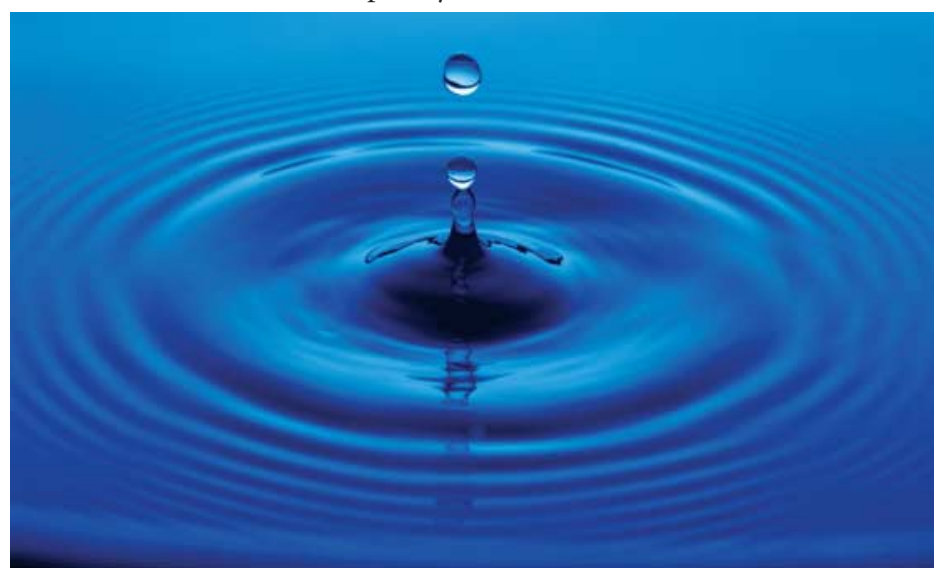

\section{IN BRIEF}

ENVIRONMENTAL MICROBIOLOGY

Role of metal-reducing bacteria in arsenic release from Bengal delta sediments

Islam, F. S. et al. Nature 430, 68-71 (2004)

The contamination of groundwater by the release of arsenic from sediments poses a major threat to human health in some parts of Asia, particularly Bangladesh and West Bengal. The mechanisms responsible for this release have been hotly debated. Now, Islam and colleagues report in Nature that their research using a laboratory microcosm approach has provided direct evidence that anaerobic metal-reducing bacteria are involved in the formation of toxic $\mathrm{As}_{\text {(III) }}$ in sediments collected in West Bengal. They also obtained data to support the theory that the introduction of carbon, for example by irrigation techniques, could be enhancing the mobility of arsenic in shallow groundwater in this area.

\section{BACTERIAL TOXINS}

A new family of potent $A B_{5}$ cytotoxins produced by Shiga toxigenic Escherichia coli

Paton, A. W. et al. J. Exp. Med. (June 28 2004) doi: 10.1084/jem.20040392

Toxins belonging to the $\mathrm{AB}_{5}$ toxin family are potent virulence factors produced by some bacterial pathogens such as Shiga toxigenic E. coli and Shigella dysenteriae (Shiga toxin), and Bordetella pertussis (pertussis toxin). $\mathrm{AB}_{5}$ toxins comprise an enzymatic A subunit and a pentameric $B$ subunit that is responsible for docking the toxin onto target eukaryotic host cells. In this work, Paton et al. characterize a new member of the $\mathrm{AB}_{5}$ toxin family from a highly virulent Shiga toxigenic E. coli strain. The A subunit of this new toxin has a subtilase-like activity, the first time that a cytotoxic effect has been associated with this serine protease family. The authors speculate that this new toxin could work synergistically with Shiga toxin in the pathogenesis of Shiga toxigenic E. coli.

\section{ENVIRONMENTAL MICROBIOLOGY}

\section{Molecular analysis of shower curtain biofilm microbes}

Kelly, S. T. et al. Appl. Environ. Microbiol. 70, 4187-4192 (2004)

When did you last clean your shower curtain? Over time, vinyl shower curtains accumulate a deposit commonly known as soap scum. In a new study published in Applied and Environmental Microbiology, Kelly et al. used a variety of techniques to analyse this material from four shower curtains that had been in use for more than 6 months. Epifluorescence microscopy demonstrated that soap scum contains a rich variety of microorganisms within a biofilm matrix. DNA from the shower curtain communities was extracted and sequenced. rRNA analysis revealed that the composition of each microbial community was complex, with Sphingomonas spp. and Methylobacterium spp. among the most prominent members. The results of this study indicate that shower curtains could be a source of opportunistic pathogens and so should be cleaned regularly, particularly in households with immunosuppressed individuals. 